Chapter 9

\title{
The Minipig - A New Tool in Stem Cell Research
}

\author{
Hideyuki Kobayashi, Toshihiro Tai, \\ Koichi Nagao and Koichi Nakajima \\ Additional information is available at the end of the chapter \\ http://dx.doi.org/10.5772/57603
}

\section{Introduction}

The establishment of pluripotent mouse embryonic stem cells (ESCs), which have the capacity to differentiate into all cell types in the mammalian body, was first described by Evans and Kaufman in 1981. Seventeen years later, in 1998, the first report of human ESCs, with similar differentiation characteristics was published by James Thompson. In 2006, Takahashi and Yamanaka discovered that the genome of a differentiated somatic cell could be epigenetically reprogrammed to pluripotency by inducing the expression of pluripotency transcription factors, resulting in the production of pluripotent stem cells (iPSCs). This research has accelerated the study of regenerative medicine.

In 2009, the US Food and Drug Administration approved the first Phase I clinical trials of human ESC-derived cells for the treatment of spinal cord injuries. However, previous reports had revealed that stem cell-based therapies increase the risk of tumor development. Therefore, further basic research is needed before stem cell-based therapies can be applied in the clinic. The development of stem cell tools can be critically evaluated using animal models that express human disease genes.

Research on the stem cell biology of the minipig is developing rapidly. Although research on mouse and human stem cells currently predominates over that in other species, data from these well-studied species have provided a good foundation for current and future porcine stem cell research. In addition, the increasing popularity of alternative-species models for the study of human diseases and disease mechanisms has further spurred porcine stem cell research. As a model system for pluripotent embryonic stem cell research, however, the pig presents several challenges as compared with mice and humans. Nonetheless, porcine minipig embryonic germ cells have recently been produced, and may prove particularly useful for in vitro and in vivo differentiation studies, gene targeting, and the creation of transgenic animals. In addition, 
studies involving the transplantation of somatic mesenchymal stem cells into porcine heart, cartilage, and bone have yielded very promising results. Lastly, minipig induced pluripotent stem cells have been established by using Sendai viruses to introduce pluripotency transcription factors into the cells.

Thus, despite the challenges of developing porcine pluripotent stem cells, recent successes in the fields of both induced pluripotent stem cells and somatic stem cells suggest that the future of research using minipig stem cells is quite promising.

\section{Embryonic stem cells}

Most porcine embryonic stem cell research has been performed in large domestic pig breeds rather than minipigs. Therefore, this discussion will focus on studies performed on the large domestic breeds, yet with attention paid to outcomes observed in the minipig. Research in the domestic species is highly transferrable to the minipig.

Although researchers have sought to establish ESCs in the pig, the characterization of ESCs in this animal falls short, due to a lack of both in vivo and long-term culture studies as compared to ESCs of mouse or human origin. The first reports on ESC production in the pig were in 1990 [1] [2] [3] [4]. However, those attempts produced only putative ESCs or embryonic stem-like cells.

A number of standard techniques are typically employed to verify the identity of true ESCs. Several in vitro techniques are generally performed to determine the expression profile of the ESCs, including gene-expression and protein analyses. The transcription factors expressed in both mouse and human ESCs include OCT4, NANOG, and SOX2. In addition, stage-specific embryonic antigen 1 (SSEA1) is expressed in mouse but not human ESCs, and SSEA3 and SSEA4 are detected in human but not mouse ESCs [5].

While the efforts to establish porcine ESCs have been well reviewed in the scientific literature [6] [7], a general overview of the research suggests that true porcine ESCs have not yet been produced. The production of porcine epiblast stem cells (EpiSCs) was recently reported [8]. These cells are thought to be derived from the epiblast, rather than the inner cell mass, of the developing embryo. Evidence suggests that mouse ESCs are of inner cell mass origin, while human ESCs originate from epiblasts [9]. The porcine epiblast stem cells could be cultured for 22 passages, and could differentiate in vitro into cell types representative of the three embryonic germ lineages as well as germ precursor cells and trophectoderm. However, it is not known whether these cells also demonstrate pluripotency.

To date, two research groups have attempted to produce porcine ESCs from the minipig [10, 11]. Li and colleagues reported that outgrowth cultures could be obtained after isolating the inner cell mass from Chinese minipig blastocysts, although sustained culture was difficult to achieve, and only a preliminary characterization of these cells was performed [10]. Long-term cultures of porcine ESC-like cells were reported by Kim and colleagues [11]. In this study, porcine ESC-like cells were derived from cloned blastocysts. These embryos were produced 
by somatic cell nuclear transfer, using minipig fetal or neonatal fibroblasts as the donor and prepubertal gilt oocytes, followed by culture in vitro to the blastocyst stage of development. Two cells lines could be cultured for more than 48 passages, and expressed alkaline phosphatase (AP), SSEA1 and SSEA3, OCT4, TRA-1-60, and TRA-1-81. The only method used to characterize their differentiation ability was the observation of spontaneous differentiation into embryoid bodies (EBs), which are spheres of cells that contain cell types of all three germ layers. These EBs were assessed solely by gene analysis, thus further characterization would be necessary before they could be verified as true ESCs.

Only two reports to date describe the production of chimeras from porcine ESC-like cells: one in 1999, from Chen and colleagues [12], and a more recent publication in 2010 [13]. The first report claimed that somatic chimeric piglets could be produced, although clear analyses of these chimeric animals was lacking. The second report indicated that porcine ESC-like cells from an early passage could form chimeric piglets. The chimeric contribution was low, however; only 4 chimeric piglets were born after the transfer of hundreds of embryos. Only 2 of the 4 chimeric piglets showed coat chimerism, and this contribution was low and restricted to a single spot near the tail. Such a chimeric contribution is much lower than what would be expected from mouse ESCs, indicating that improvements to the cell culture conditions may be required to improve the plasticity of these cells.

There are several possible reasons for the difficulty in producing porcine ESCs. The lack of defined culture conditions may be one reason. Pluripotency appears to be controlled by more than one cell-signaling pathway, and these pathways are different in mouse and human ESC lines. The origin of the cells, that is, the inner cell mass (mouse) versus the epiblasts (human) may contribute to this diversity of regulatory pathways. This idea is supported by a recent publication showing that even mouse EpiSCs regulate pluripotency slightly differently from human ESCs [14]. The cell signaling that governs pluripotency in the pig remains largely unknown, although the details are beginning to be investigated [15]. For example, it was reported that fibroblast growth factor (FGF) signaling may be active in porcine epiblasts, and that the JAK/STAT pathway is inactive. The Activin/Nodal pathway also appears to be active in porcine epiblasts [8]. Culturing porcine epiblasts in medium containing basic FGF (bFGF) cannot prevent their differentiation, indicating that other factors are apparently necessary to help maintain cellular pluripotency.

Differences occurring during early embryonic development in the domestic pig, as compared with mouse and human, could also account for the observed difficulties in producing porcine ESCs. The early development of the porcine embryo prior to implantation takes longer and is less advanced than in the other species. The inner cell mass differentiates into the hypoblast and epiblast at a later time point than in the mouse and human, and the porcine epiblast expands and develops over a period of several days. The cell signaling controlling this development in the pig could differ markedly from that in the mouse or human, and should be investigated to ascertain which stage of development is optional for isolating the pluripotent cells. It is possible that the later epiblast is already predetermined at the cell-signaling level to undergo gastrulation, or that the inner cell mass cells have not yet acquired the necessary cell signaling machinery to support proliferation. 
We have yet to observe bona fide ESCs produced from the pig. Nevertheless, continued research toward this goal is important, as the pig is a particularly useful biomedical model for studying human disease, and ESCs are a unique cell type that is especially useful for studying human disease.

\section{Embryonic germ cells}

Embryonic germ cells (EGCs) are pluripotent cells derived in vitro from primordial germ cells (PGCs). They were first derived in the mouse by Matsui et al. [16] and Resnick et al. [17]. Mouse EGCs are morphologically similar to mouse ESCs, and express similar pluripotency markers such as AP, SSEA1, Oct4, Sox2, and Nanog. The differentiation potential of these cells is also similar to that of mouse ESCs, as evidenced by their ability to differentiate in vitro into different tissues from the three primary germ layers [18], as well as to re-enter the germline when injected into blastocyst embryos [19]. Mouse EGCs may thus be considered equivalent to ESCs.

Similar to the conditions required to derive mouse ESCs, mouse EGCs need two important growth factors in addition to leukemia inhibitory factor (LIF) for their successful derivation from PGCs. The first is stem cell factor (SCF), a ligand for c-Kit, which supports PGC survival in vivo and in vitro by suppressing apoptosis [20]. The second is bFGF, which plays a major role in the reprogramming of mouse PGCs into pluripotent cells in vitro [21]. Although the mechanism of this reprogramming is still poorly understood, bFGF is known to downregulate the expression of basic lymphocyte maturation protein 1 (Blimp1) in vitro, which in turn causes the upregulation of c-Myc and Klf4 [21]. Because mouse PGCs also express Oct4 and Sox2, a similar mechanism may be involved in their reprogramming to ESCs.

Porcine EGCs were first derived by Shim et al. [22], who used embryos from domestic breeds on day 25 of gestation under conditions similar to those used to culture mouse EGCs. The established cell lines had an ESC-like morphology, expressed AP, and were able to differentiate into different cell types in vitro and in vivo. Other groups have derived porcine EGC lines using day 25-28 embryos from domestic breeds [23] [24] [25] [26] [27], and from the Chinese minipig [28]. Recently, putative porcine EGC lines derived from the PGCs of day 20-24 embryos from Danish Landrace crosses and Yucatan minipigs were reported [29]. Notably, these findings suggest that EGC lines can be established from any porcine breed, unlike mouse ESCs, which are restricted to certain strains.

Immunocytochemical analysis of pluripotency marker expression showed that, in addition to AP activity, EGCs express Oct4, SSEA1, SSEA3, and SSEA4 at variable levels. Analysis of the porcine EGC gene expression suggested that they are similar to human EGCs in expressing AP, Oct4, SSEA1, and SSEA4 [30]. The pig EGCs typically have long cell cycles, and proliferate slowly over many passages.

The tissue culture conditions used by all groups for the derivation and propagation of porcine EGCs are similar to those used for human and mouse ESCs. The cells are grown on feeder layers of mitotically inactivated embryonic mouse fibroblasts, most often on immortalized 
mouse fibroblast cells from a cell line known as STO. The initial establishment of cell lines is hampered by the fact that large numbers of porcine PGCs undergo apoptosis and die in culture within 6 hours of their incubation [25]. However, by using protease inhibitors and antioxidants to reduce the level of PGC apoptosis in vitro, Lee et al. were able to increase the number of EGC colonies in the primary culture. In addition, alpha 2-macroglobulin together with N-acetylcystein and butylated hydroxyanisole (both antioxidants) increased the number of AP-positive colonies in primary cultures at least twofold. Another approach is to use growth factors and various feeder cells to increase the survival and prevent the differentiation of PGCs in longterm culture. Lee et al. [26] showed that supplementing the culture medium with three growth factors (LIF, SCF, and bFGF) increased the number of colonies obtained in primary culture, and improved the quality of the colonies in subsequent passages compared to cells treated with only two of these growth factors. The use of feeder layers other than STO cells did not have a significant effect on PGC survival or on the quality of the derived EGCs. In another study, membrane-bound SCF and soluble LIF were sufficient to increase the number of surviving PGCs on days 3 and 5 of primary culture, while adding bFGF did not affect the results significantly [23]. It is possible that the membrane-bound form of SCF is more potent than the soluble form, which could explain these differing results. However, it has also been reported that established PGCs are capable of generating chimeras without added growth factors, while adding LIF to the culture does not improve the efficiency of establishing EGC lines [22]. Thus, further study is needed to elucidate the effects of these supplements in culture.

Pluripotent stem cells are able to form EBs, spherical aggregates containing differentiated cells, when cultured in suspension. Porcine EGCs cultured in "hanging drops" can also form simple EBs, consisting of large epithelial-like cells on the periphery surrounding mesenchymal-like cells in the center. When allowed to attach to gelatin-treated plastic dishes, the EB cells proliferate and spread on the surface of the dish, giving rise to several different types of cells [27].

Pluripotent cells can proliferate and differentiate in vivo, forming tumors containing differentiated tissues called teratomas, when injected into immunodeficient mice. To date, only one group has reported teratoma testing for porcine EGCs, using cell lines derived from the Chinese minipig [28]. The authors reported that the teratomas contained cells from the three primary germ layers: epithelial, neuroepithelial, and adipose tissue. In contrast to these results, two studies showed that the injection of human EGCs into immunodeficient mice failed to generate teratomas. [31] [32]. However, more recently, the formation of teratomas from human EGCs cultured under serum-free conditions was reported [33].

Another way of testing the differentiation potential of pluripotent cells in vivo is by chimera formation, in which the cells injected into early embryos contribute to the three germ layers and potentially to the germline. Unlike mouse ESCs and EGCs, which have been shown to reenter the germline of chimeras, pig EGC chimeras have not displayed a proven germline contribution; furthermore, the somatic tissues of these porcine chimeras contained only a low percentage of donor-derived cells [22] [34] [24]. Other researchers demonstrated a similarly low chimeric contribution after the injection of somatic cells into sheep blastocysts and 8-cell mouse embryos $[35,36]$. This is troubling because germline contribution is considered to be 
the ultimate proof of pluripotency, and has been demonstrated only in the mouse and chicken to date. Human ESCs are presumed to be true stem cells as well, even though their germline potential has not been tested for ethical reasons. Caution is needed when interpreting chimera experimental results. Proper controls are necessary to distinguish the "true" stem cells from somatic cells that can be integrated into the embryos after partial reprogramming by the surrounding embryonic cells.

The reasons for the limited pluripotency of the porcine EGCs are currently unclear. It is possible that the tissue culture conditions are not sufficient for maintaining the pluripotency of the PGCs in vitro, similar to the problems that exist in the cultures of inner cell mass and epiblast cells. Another possibility is that the pig PGCs do not undergo full epigenetic reprogramming into pluripotency like the mouse PGCs do. One indication that there might be differences in the biology of PGCs between the pig and mouse is that porcine PGCs survive and proliferate in vitro in the absence of externally added growth factors, regardless of whether they are cultured in serum-supplemented or serum-free conditions, while mouse PGCs fail to survive in the absence of any of the three growth factors LIF, SCF, or bFGF. In addition, mouse PGCs have not been shown to form chimeras, whereas porcine PGCs can contribute to somatic tissues after their injection into early blastocysts [24]. Thus, some of the molecular mechanisms that are important for reprogramming mouse PGCs to pluripotent EGCs in culture may be different in the pig.

\section{Induced pluripotent stem cells}

Takahashi and Yamanaka [37] discovered that the genome of a differentiated somatic cell can be epigenetically reprogrammed to pluripotency by the induced expression of pluripotency transcription factors, resulting in the generation of pluripotent stem cells [induced Pluripotent Stem Cells (iPSCs)]. These authors initially expressed 24 pluripotency genes in fetal fibroblasts to reprogram them into pluripotent cells, and subsequently found that the expression of only 4 of these genes, namely Oct4, Sox2, c-myc, and Klf4, was sufficient to achieve the same results. Nanog was dispensable for the cellular reprogramming. In addition, a year later, Okita et al. [38] produced germline-competent mouse iPSCs using the same growth factors, but by selecting the reprogrammed cells by Nanog expression.

The production of human iPSCs started shortly after the publication of the first report of mouse iPSC derivation. Thus, it was not surprising that the establishment of the first human iPSCs was reported by two groups simultaneously. The laboratory that produced the first mouse iPSCs successfully generated iPSCs from adult human fibroblasts by using the same 4 transcription factors employed in the initial experiment [39]. The other group, from the laboratory of human ESC pioneer James Thompson, was able to reprogram human fibroblasts by expressing OCT4, SOX2, NANOG, and LIN28 [40].

Predictably, these studies sparked significant interest among researchers working in the area of porcine pluripotent cells. Within two years after the publication of the first papers describing 
human and mouse iPSCs, three different groups reported the establishment of iPSCs in the pig. One of these groups generated porcine iPSCs using fetal fibroblasts from Danish Landrace pigs, by the lentiviral transduction of six human transcription factors (OCT4, SOX2, c-Myc, KLF4, LIN28, and NANOG) under the control of a doxycycline-inducible promoter [41]. Simultaneously, another Chinese group reported the derivation of iPSCs from fibroblasts from the Tibetan minipig, using constitutively expressed lentiviral vectors carrying the mouse cDNA sequences of Oct4, Sox2, c-myc, and Klf4 [42]. The third report published shortly thereafter by Ezashi et al. [43] also described the production of porcine iPSCs through the use of 4 human transcription factors (OCT4, SOX2, c-Myc, and KLF4). In addition, these researchers claimed that the porcine iPSCs were positive for SSEA-1 but negative for SSEA3 and SSEA-4, indicating that they were more similar to mouse than human iPSCs.

Transcriptional profiling of the cell line by Affymetrix microarray confirmed that the cells were indeed reprogrammed, and expressed a variety of ESC markers endogenously. However, the continued expression of the exogenous transcription factors was detected in the iPSCs generated by all three groups. This problem is not unique to the pig, as it has been reported in other species as well [39]. In any case, the continued expression of pluripotency genes did not pose any problems for the differentiation of the cells, since all three laboratories demonstrated that their cell lines were able to differentiate in vitro (including EB formation) and in vivo by forming teratomas containing cells of all three germ layers. More recently, it was demonstrated that porcine iPSCs can form chimeras with high efficiency (85.3\%), and contribute to all three germ layers [44]. Because germline chimerism was not confirmed in this study, it remains to be determined whether the porcine iPSCs are fully equivalent to mouse iPSCs.

One of the major advantages iPSCs may offer in the future is the potential for custom derivation of pluripotent cells from individual patients to use for regenerative therapies without the risk of immune rejection. However, since the epigenetic reprogramming necessary to produce them requires prolonged expression of the transgenes (2-3 weeks), most of the iPSCs described to date have been generated with the use of lentiviral vectors that integrate known oncogenes (such as c-myc and Klf4) into the cell genome. The dangers of using these genes became evident in a study in which mouse chimeras generated with iPSCs developed tumors following reactivation of the initially silenced transgene c-myc [38]. In the only report to date in which porcine chimeras were produced from iPSCs [44], no tumor formation was reported in the newborn piglets. However, because long-term testing and monitoring were not conducted prior to the publication of these results, at present there is no guarantee that these iPSCs would be safe for clinical applications.

To avoid problems associated with viral integration and the use of oncogenes, many groups have developed strategies for iPSC production in which the disadvantages discussed above have been minimized. For example, it has been shown that reprogramming can be achieved in both human and mouse cells without the use of c-myc, albeit at the expense of efficiency [45]. In addition, the use of certain transcription factors can be omitted when using cell types already expressing them; for example, neural progenitor cells, which already express endogenous Sox 2 and c-myc were reprogrammed using only the induced expression of OCT4 and Klf4 [46] [47]. 
Furthermore, some transcription factors can be replaced by small molecules that have similar effects on somatic cell reprogramming [48]. Viral integration, which carries risks of insertional mutagenesis, can be avoided by using nonintegrating adenoviral vectors [49] [50]. Our laboratory has established porcine iPSCs derived from pig skin fibroblasts using Sendai virus vectors to introduce 4 human transcription factors (OCT4, SOX2, c-Myc, and KLF4) (data not shown). Another attractive alternative to the use of viral vectors are transposon vectors, which combine high transfection efficiency with enhanced safety, and can be removed from the cell genomes following reprogramming. For example, piggyBac is a DNA transposon that, following insertion, can be removed from the reprogrammed genome without leaving a trace. This transposon has already been used successfully to produce iPSCs [51, 52]. Finally, Zhou [53] produced iPSCs by providing the transcription factors in the form of recombinant proteins.

With regard to future research in porcine iPSC production, the choice of cell type and strategy will undoubtedly play an important role in the reprogramming efficiency. It should be noted, however, that the same problems existing in porcine ESC culture are likely to affect the longterm maintenance of the newly generated pig iPSC lines. For example, the culture conditions supporting the pluripotency and self-renewal of the porcine iPSCs apparently require further optimization, given that, in all the published reports to date, the reprogrammed cell lines could be maintained only with the continued expression of exogenous pluripotency genes. In one study, for example, instead of using growth factors or other supplements, the pig iPSCs were maintained with doxycycline-induced expression of the pluripotency transgenes, until the authors chose to differentiate the cells [41].

Despite these challenges, we can without doubt look forward to an exciting future in which iPSCs will add another dimension to pluripotent stem cell research in the pig.

\section{Epiblast stem cells}

Epiblast stem cells are pluripotent stem cells derived from the epiblast layer of post-implantation mouse embryos [54]. Epiblast stem cells are distinct from ES cells, which are derived from the inner cell mass of blastocysts. Both ES cells and epiblast stem cells can differentiate into mesoderm, endoderm, and ectoderm. However, epiblast stem cells do not have germ-line transmission.

Rodent pluripotent stem cells are considered to have two distinct states: naïve and primed. Naïve pluripotent stem cell lines are distinguished from primed cells in their response to LIF signaling and MEK/GSK3 inhibition (LIF/2i conditions) and X chromosome activation. Human ES and iPS cells both resemble rodent primed epiblast stem cells more closely than rodent naïve ES cells. In addition, iPS cells derived from pigs can obtain the properties of primed epiblast stem cells. iPS cells derived from human, monkey, rabbit, and rat, but not mouse, can also obtain the properties of primed epiblast stem cells [55]. 


\section{Conclusions}

Stem cell biology research of the minipig is developing rapidly. Although studies on mouse and human stem cells currently outnumber those of other species, data from these well-studied species provide a good foundation for current and future porcine stem cell research. Despite the challenges associated with developing porcine pluripotent stem cells, recent successes in the fields of induced pluripotent stem cells and somatic stem cells suggest that minipig stem cell research has a promising future.

\section{Acknowledgements}

This study was supported in part by a Grant-in-Aid for Young Scientists (B) of the Japan Society for the Promotion of Science (JSPS) and Grant of Strategic Research Foundation Grant-aided Project for Private schools at Heisei 23th from Ministry of Education, Culture, Sports, Science and Technology of Japan, 2011-2015.

\section{Author details}

Hideyuki Kobayashi*, Toshihiro Tai, Koichi Nagao and Koichi Nakajima

*Address all correspondence to: hideyukk@med.toho-u.ac.jp

Department of Urology, Toho University School of Medicine, Tokyo, Japan

\section{References}

[1] Notarianni E, Laurie S, Moor RM, Evans MJ. Maintenance and differentiation in culture of pluripotential embryonic cell lines from pig blastocysts. J Reprod Fertil Suppl. 1990;41:51-6.

[2] Piedrahita JA, Anderson GB, Bondurant RH. On the isolation of embryonic stem cells: Comparative behavior of murine, porcine and ovine embryos. Theriogenology. 1990;34(5):879-901.

[3] Piedrahita JA, Anderson GB, Bondurant RH. Influence of feeder layer type on the efficiency of isolation of porcine embryo-derived cell lines. Theriogenology. 1990;34(5): 865-77. 
[4] Strojek RM, Reed MA, Hoover JL, Wagner TE. A method for cultivating morphologically undifferentiated embryonic stem cells from porcine blastocysts. Theriogenology. 1990;33(4):901-13.

[5] Henderson JK, Draper JS, Baillie HS, Fishel S, Thomson JA, Moore H, et al. Preimplantation human embryos and embryonic stem cells show comparable expression of stage-specific embryonic antigens. Stem Cells. 2002;20(4):329-37.

[6] Hall V. Porcine embryonic stem cells: a possible source for cell replacement therapy. Stem Cell Rev. 2008;4(4):275-82.

[7] Oestrup O, Hall V, Petkov SG, Wolf XA, Hyldig S, Hyttel P. From zygote to implantation: morphological and molecular dynamics during embryo development in the pig. Reprod Domest Anim. 2009;44 Suppl 3:39-49.

[8] Alberio R, Croxall N, Allegrucci C. Pig epiblast stem cells depend on activin/nodal signaling for pluripotency and self-renewal. Stem Cells Dev. 2010;19(10):1627-36.

[9] Reijo Pera RA, DeJonge C, Bossert N, Yao M, Hwa Yang JY, Asadi NB, et al. Gene expression profiles of human inner cell mass cells and embryonic stem cells. Differentiation. 2009;78(1):18-23.

[10] Li M, Ma W, Hou Y, Sun XF, Sun QY, Wang WH. Improved isolation and culture of embryonic stem cells from Chinese miniature pig. J Reprod Dev. 2004;50(2):237-44.

[11] Kim S, Kim JH, Lee E, Jeong YW, Hossein MS, Park SM, et al. Establishment and characterization of embryonic stem-like cells from porcine somatic cell nuclear transfer blastocysts. Zygote. 2010;18(2):93-101.

[12] Chen LR, Shiue YL, Bertolini L, Medrano JF, BonDurant RH, Anderson GB. Establishment of pluripotent cell lines from porcine preimplantation embryos. Theriogenology. 1999;52(2):195-212.

[13] Vassiliev I, Vassilieva S, Beebe LF, Harrison SJ, McIlfatrick SM, Nottle MB. In vitro and in vivo characterization of putative porcine embryonic stem cells. Cell Reprogram. 2010;12(2):223-30.

[14] Greber B, Wu G, Bernemann C, Joo JY, Han DW, Ko K, et al. Conserved and divergent roles of FGF signaling in mouse epiblast stem cells and human embryonic stem cells. Cell Stem Cell. 2010;6(3):215-26.

[15] Hall VJ, Christensen J, Gao Y, Schmidt MH, Hyttel P. Porcine pluripotency cell signaling develops from the inner cell mass to the epiblast during early development. Dev Dyn. 2009;238(8):2014-24.

[16] Matsui Y, Zsebo K, Hogan BL. Derivation of pluripotential embryonic stem cells from murine primordial germ cells in culture. Cell. 1992;70(5):841-7.

[17] Resnick JL, Bixler LS, Cheng L, Donovan PJ. Long-term proliferation of mouse primordial germ cells in culture. Nature. 1992;359(6395):550-1. 
[18] Rohwedel J, Sehlmeyer U, Shan J, Meister A, Wobus AM. Primordial germ cell-derived mouse embryonic germ (EG) cells in vitro resemble undifferentiated stem cells with respect to differentiation capacity and cell cycle distribution. Cell Biol Int. 1996;20(8):579-87.

[19] Labosky PA, Barlow DP, Hogan BL. Mouse embryonic germ (EG) cell lines: transmission through the germline and differences in the methylation imprint of insulin-like growth factor 2 receptor (Igf2r) gene compared with embryonic stem (ES) cell lines. Development. 1994;120(11):3197-204.

[20] Pesce M, Farrace MG, Piacentini M, Dolci S, De Felici M. Stem cell factor and leukemia inhibitory factor promote primordial germ cell survival by suppressing programmed cell death (apoptosis). Development. 1993;118(4):1089-94.

[21] Durcova-Hills G, Tang F, Doody G, Tooze R, Surani MA. Reprogramming primordial germ cells into pluripotent stem cells. PLoS One. 2008;3(10):e3531.

[22] Shim H, Gutiérrez-Adán A, Chen LR, BonDurant RH, Behboodi E, Anderson GB. Isolation of pluripotent stem cells from cultured porcine primordial germ cells. Biol Reprod. 1997;57(5):1089-95.

[23] Durcova-Hills G, Prelle K, Müller S, Stojkovic M, Motlik J, Wolf E, et al. Primary culture of porcine PGCs requires LIF and porcine membrane-bound stem cell factor. Zygote. 1998;6(3):271-5.

[24] Mueller S, Prelle K, Rieger N, Petznek H, Lassnig C, Luksch U, et al. Chimeric pigs following blastocyst injection of transgenic porcine primordial germ cells. Mol Reprod Dev. 1999;54(3):244-54.

[25] Lee CK, Piedrahita JA. Effects of growth factors and feeder cells on porcine primordial germ cells in vitro. Cloning. 2000;2(4):197-205.

[26] Lee CK, Weaks RL, Johnson GA, Bazer FW, Piedrahita JA. Effects of protease inhibitors and antioxidants on In vitro survival of porcine primordial germ cells. Biol Reprod. 2000;63(3):887-97.

[27] Petkov SG, Anderson GB. Culture of porcine embryonic germ cells in serum-supplemented and serum-free conditions: the effects of serum and growth factors on primary and long-term culture. Cloning Stem Cells. 2008;10(2):263-76.

[28] Tsung HC, Du ZW, Rui R, Li XL, Bao LP, Wu J, et al. The culture and establishment of embryonic germ (EG) cell lines from Chinese mini swine. Cell Res. 2003;13(3): 195-202.

[29] Petkov SG, Marks H, Klein T, Garcia RS, Gao Y, Stunnenberg H, et al. In vitro culture and characterization of putative porcine embryonic germ cells derived from domestic breeds and Yucatan mini pig embryos at Days 20-24 of gestation. Stem Cell Res. 2011;6(3):226-37. 
[30] Turnpenny L, Brickwood S, Spalluto CM, Piper K, Cameron IT, Wilson DI, et al. Derivation of human embryonic germ cells: an alternative source of pluripotent stem cells. Stem Cells. 2003;21(5):598-609.

[31] Shamblott MJ, Axelman J, Littlefield JW, Blumenthal PD, Huggins GR, Cui Y, et al. Human embryonic germ cell derivatives express a broad range of developmentally distinct markers and proliferate extensively in vitro. Proc Natl Acad Sci U S A. 2001;98(1):113-8.

[32] Turnpenny L, Cameron IT, Spalluto CM, Hanley KP, Wilson DI, Hanley NA. Human embryonic germ cells for future neuronal replacement therapy. Brain Res Bull. 2005;68(1-2):76-82.

[33] Hua J, Yu H, Liu S, Dou Z, Sun Y, Jing X, et al. Derivation and characterization of human embryonic germ cells: serum-free culture and differentiation potential. Reprod Biomed Online. 2009;19(2):238-49.

[34] Piedrahita JA, Moore K, Oetama B, Lee CK, Scales N, Ramsoondar J, et al. Generation of transgenic porcine chimeras using primordial germ cell-derived colonies. Biol Reprod. 1998;58(5):1321-9.

[35] Karasiewicz J, Sacharczuk M, Was B, Guszkiewicz A, Korwin-Kossakowski M, Gorniewska $\mathrm{M}$, et al. Experimental embryonic-somatic chimaerism in the sheep confirmed by random amplified polymorphic DNA assay. Int J Dev Biol. 2008;52(2-3): $315-22$.

[36] Piliszek A, Modliński JA, Pyśniak K, Karasiewicz J. Foetal fibroblasts introduced to cleaving mouse embryos contribute to full-term development. Reproduction. 2007;133(1):207-18.

[37] Takahashi K, Yamanaka S. Induction of pluripotent stem cells from mouse embryonic and adult fibroblast cultures by defined factors. Cell. 2006;126(4):663-76.

[38] Okita K, Ichisaka T, Yamanaka S. Generation of germline-competent induced pluripotent stem cells. Nature. 2007;448(7151):313-7.

[39] Takahashi K, Tanabe K, Ohnuki M, Narita M, Ichisaka T, Tomoda K, et al. Induction of pluripotent stem cells from adult human fibroblasts by defined factors. Cell. 2007;131(5):861-72.

[40] Yu J, Vodyanik MA, Smuga-Otto K, Antosiewicz-Bourget J, Frane JL, Tian S, et al. Induced pluripotent stem cell lines derived from human somatic cells. Science. 2007;318(5858):1917-20.

[41] Wu Z, Chen J, Ren J, Bao L, Liao J, Cui C, et al. Generation of pig induced pluripotent stem cells with a drug-inducible system. J Mol Cell Biol. 2009;1(1):46-54. 
[42] Esteban MA, Xu J, Yang J, Peng M, Qin D, Li W, et al. Generation of induced pluripotent stem cell lines from Tibetan miniature pig. The Journal of biological chemistry. 2009;284(26):17634-40.

[43] Ezashi T, Telugu BP, Alexenko AP, Sachdev S, Sinha S, Roberts RM. Derivation of induced pluripotent stem cells from pig somatic cells. Proc Natl Acad Sci U S A. 2009;106(27):10993-8.

[44] West FD, Terlouw SL, Kwon DJ, Mumaw JL, Dhara SK, Hasneen K, et al. Porcine induced pluripotent stem cells produce chimeric offspring. Stem Cells Dev. 2010;19(8): 1211-20.

[45] Nakagawa M, Koyanagi M, Tanabe K, Takahashi K, Ichisaka T, Aoi T, et al. Generation of induced pluripotent stem cells without Myc from mouse and human fibroblasts. Nat Biotechnol. 2008;26(1):101-6.

[46] Kim JB, Zaehres H, Wu G, Gentile L, Ko K, Sebastiano V, et al. Pluripotent stem cells induced from adult neural stem cells by reprogramming with two factors. Nature. 2008;454(7204):646-50.

[47] Shi Y, Desponts C, Do JT, Hahm HS, Schöler HR, Ding S. Induction of pluripotent stem cells from mouse embryonic fibroblasts by Oct4 and Klf4 with small-molecule compounds. Cell Stem Cell. 2008;3(5):568-74.

[48] Lin T, Ambasudhan R, Yuan X, Li W, Hilcove S, Abujarour R, et al. A chemical platform for improved induction of human iPSCs. Nat Methods. 2009;6(11):805-8.

[49] Okita K, Nakagawa M, Hyenjong H, Ichisaka T, Yamanaka S. Generation of mouse induced pluripotent stem cells without viral vectors. Science. 2008;322(5903):949-53.

[50] Stadtfeld M, Nagaya M, Utikal J, Weir G, Hochedlinger K. Induced pluripotent stem cells generated without viral integration. Science. 2008;322(5903):945-9.

[51] Woltjen K, Michael IP, Mohseni P, Desai R, Mileikovsky M, Hämäläinen R, et al. piggyBac transposition reprograms fibroblasts to induced pluripotent stem cells. Nature. 2009;458(7239):766-70.

[52] Yusa K, Rad R, Takeda J, Bradley A. Generation of transgene-free induced pluripotent mouse stem cells by the piggyBac transposon. Nat Methods. 2009;6(5):363-9.

[53] Zhou H, Wu S, Joo JY, Zhu S, Han DW, Lin T, et al. Generation of induced pluripotent stem cells using recombinant proteins. Cell Stem Cell. 2009;4(5):381-4.

[54] Brons IG, Smithers LE, Trotter MW, Rugg-Gunn P, Sun B, Chuva de Sousa Lopes $\mathrm{SM}$, et al. Derivation of pluripotent epiblast stem cells from mammalian embryos. Nature. 2007;448(7150):191-5.

[55] De Los Angeles A, Loh YH, Tesar PJ, Daley GQ. Accessing naive human pluripotency. Current opinion in genetics \& development. 2012;22(3):272-82. 
